\title{
Fabrication tolerant design of silicon nitride Kerr comb generators
}

Comis, Farah, Ruocco, Alfonso

Farah Comis, Alfonso Ruocco, "Fabrication tolerant design of silicon nitride Kerr comb generators," Proc. SPIE 11880, Emerging Applications in Silicon Photonics II, 118800F (6 October 2021); doi: 10.1117/12.2601583

SPIE. Event: SPIE Photonex, 2021, Glasgow, Scotland, United Kingdom 


\title{
Fabrication tolerant design of silicon nitride Kerr comb generators
}

\author{
Farah Comis ${ }^{\mathrm{a}, \mathrm{b}}$ and Alfonso Ruocco ${ }^{\mathrm{b}}$ \\ ${ }^{a}$ Electrical Division, Department of Engineering, University of Cambridge, Cambridge, CB3 \\ 0FA, UK \\ ${ }^{\mathrm{b}}$ Optical Networks Group, Department of Electronic and Electrical Engineering, University \\ College London, London, WC1E 7JE, UK
}

\begin{abstract}
We propose a solution to implement a simulation routine suitable for the design of fabrication-tolerant Kerrcomb generators by looking at the waveguides' geometry affected by the tolerance. The multiparameter-space analysis highlighted that while several waveguide cross-sections are suitable for the comb generation, they don't all provide the same safety buffer toward the fabrication variability. Thus, some designs are preferred to other suitable ones. This approach paves the way to high yield, scalable and fabrication-tolerant integrated Kerr comb generators (KCGs) manufactured in complementary metal-oxide-semiconductor (CMOS) foundries.
\end{abstract}

Keywords: Kerr comb generation, silicon nitride, integrated photonics, fabrication, nanophotonics

\section{INTRODUCTION}

In the past decades, silicon photonics has emerged as one of the most promising integration technologies thanks to several reasons, among these, its high-index contrast and its wide transparency window. ${ }^{1}$ Silicon photonic integrated circuits (PICs) offer tantalising prospects of realising many applications such as metrology, spectroscopy and telecommunications. The subwavelength regime enabled components' miniaturisation which are monolithically integrated on-chip. Consequently, they are susceptible to fabrication variability and non-uniformity, leading to unwanted mismatches between the expected and the measured performance. ${ }^{2}$ Microring resonator Kerr-comb generators implemented on high-index contrast platforms, specifically silicon nitride ( $\mathrm{SiN}$ ), enable comb generation. Subsequently, the waveguides require dispersion engineering, carried out by simulation techniques. We simulate several waveguide widths, with commonly utilised thicknesses ${ }^{3-5}$ to meet required dispersion properties. However, the fabrication variability on the KCG remains unexplored due to the computational effort required when exploring the large parameter space during the simulation phase. The computational effort can be reduced if the fabrication variations are known and stable: this is the scenario where silicon photonics is considered for future commercialisation and mass production. Through the maturity of the process, hence the stability of the fabrication variability, the computational effort can be faced only once, carrying out a proper tolerance and yield analysis. This approach will also promote re-usability for the users (customers) of the yield models associated with the platforms and the devices. The sub-micron feature size of the microring KCGs yields large variability when the fabrication is carried out with scalable techniques. ${ }^{6}$ The variability in the fabrication can be directly translated into statistical distributions of the waveguide widths and heights.

Furthermore, when partial etches are implemented, for instance when a ridge rather than a strip cross-section waveguide is utilised, the height of the ridge is affected by etching variances ${ }^{6}$ which are in general larger than the the once of the starting thin-film on the insulator. The same applies to linewidths, that are affected by patterning processes, where effects of proximity during optical lithography can induce changes. This is of concern especially when it comes to the coupling section of the microring resonator. The sensitivity of the fabrication variability

Further author information: (Send correspondence to Farah Comis)

Farah Comis: E-mail: zceefco@ucl.ac.uk

Alfonso Ruocco: E-mail: a.ruocco@ucl.ac.uk

Emerging Applications in Silicon Photonics II, edited by Callum G. Littlejohns, Marc Sorel, Proc. of SPIE Vol. 11880, 118800F · C 2021 SPIE · CCC code: 0277-786X/21/\$21 · doi: 10.1117/12.2601583 
arises from the high-index contrast nature of the structures, such as nonlinear devices. During KCG simulations, this aspect is often not thoroughly investigated, further leading to a reduced yield, which often is not a concern in academic scopes. However, the advent of silicon photonics has lead to a symbiosis between academia and industry, both often looking for ways to increase yield and improve device design robustness. For this reason, it is important for them to have a way to quantify how reliable and robust their design is before fabrication, further enabling long-term cost reduction. This work presents a simulation routine suitable for the design of fabrication tolerant KCGs. It involves the model of a multiparameter space of the SiN waveguide, by using mode solvers, and a KCG simulation tool, utilising a Lugiato-Lefever solver. ${ }^{7}$ A correlation between the tolerances associated with a specific fabrication foundry/process and the simulated performances allowed us to identify the waveguide dimensions with efficient soliton formation. We looked at the main parameters affected by the fabrication variability: the linewidth variability induced by lithography and the ridge height induced by by etching, in the first instance.

\section{COMB GENERATION BASED ON KERR-COMB GENERATION}

In order to generate optical frequency combs and achieve soliton mode-locking in microring resonators, the resonators are pumped by a single-frequency continuous wave source. The excitation of dissipative Kerr solitons (DKSs) in these rings can be achieved by tuning the frequency and/or the amplitude of the pump field. This has opened the possibility to generate fully coherent broadband combs. The Lugiato-Lefever equation (LLE) describes the dynamics of the comb generation in these microring resonators. ${ }^{8,9}$ The soliton condition in the microring resonator is described by a balance between the nonlinear length and the dispersion length. A microring with a high $\mathrm{Q}$ factor and a broadband anomalous dispersion is desired to effectively achieve these Kerr frequency combs. ${ }^{4,8}$ On one hand, a high $\mathrm{Q}$ factor ensures that the optical losses originating from the microring can be overcome by the four-wave mixing (FWM) phenomenon. The nonlinear response originating from the high intensity pump, that is the self- and cross-phase modulation, is compensated by having anomalous dispersion. ${ }^{10}$

Figure 1a illustrates the plot of the effective index of silicon nitride as bulk and as a waveguide clad with $\mathrm{SiO}_{2}$, for various cross-sections, as well as the theoretically predicted intensity distributions at the transverse electric (TE) fundamental mode at different wavelengths. It shows the effective index of the SiN waveguide approaching the index of the silicon dioxide bulk since the mode of the light leaks out into the oxide layer at higher wavelengths, while the opposite is true at shorter wavelengths when the confinement factor is higher. This behaviour is illustrated in the intensity distributions. Then, Figure 1b illustrates the SiN waveguide cross-section and the microring resonator under investigation in the simulation. The structure has a top waveguide width, denoted $w_{w}$, which spans from 600 to $2600 \mathrm{~nm}$. Then, it has a ridge height, denoted $r_{h}$, varying from 200 to $900 \mathrm{~nm}$, and finally a set of commonly used thicknesses: 300, 600 and $900 \mathrm{~nm} .^{3-5}$ Then, for the analysis of the results, the $900 \mathrm{~nm}$ thickness is selected as it displayed the most solitons and robust results.

\section{TOLERANT KCG DESIGNS}

The sub-micron feature size of the waveguides enables large scale integration. ${ }^{8}$ However, this revolutionary capability comes at a cost: the performance and the yield of the wafer scale fabricated integrated photonic devices are highly dependent on the variability associated with the fabrication utilised for their fabrication. ${ }^{2,11}$ Several measures have been explored to mitigate the fabrication variability influence on the performance and yield of the PICs and devices. Most of them require a radical novel engineering approach to the design of the circuits and their elementary building blocks. ${ }^{12}$ This approach has been proven to be successful in several examples reported in literature. ${ }^{12,13}$

The technique requires the complex photonic circuit to be broken down in elementary blocks and for each of them, mitigate the performance variability, such that the PIC will have a higher yield as a results of the individual elements increased tolerance. The validation of this approach goes through the design and testing of the sub-blocks and the wafer in several points of the article, in order to assess the actual influence of the global and local source of variability. ${ }^{1}$ This methodology often fails when the performance of the components are associated 
or depend on the dispersion properties of the waveguide used for its implementation. This is indeed the case of nonlinear devices and KCGs where the dispersion of the waveguides plays a key role in their performance. Furthermore, the validation procedure adopted for the 'bottom-up' methodology is of difficult implementation due to the size of the KCG which can not be broken down in elementary sub-blocks. ${ }^{14}$

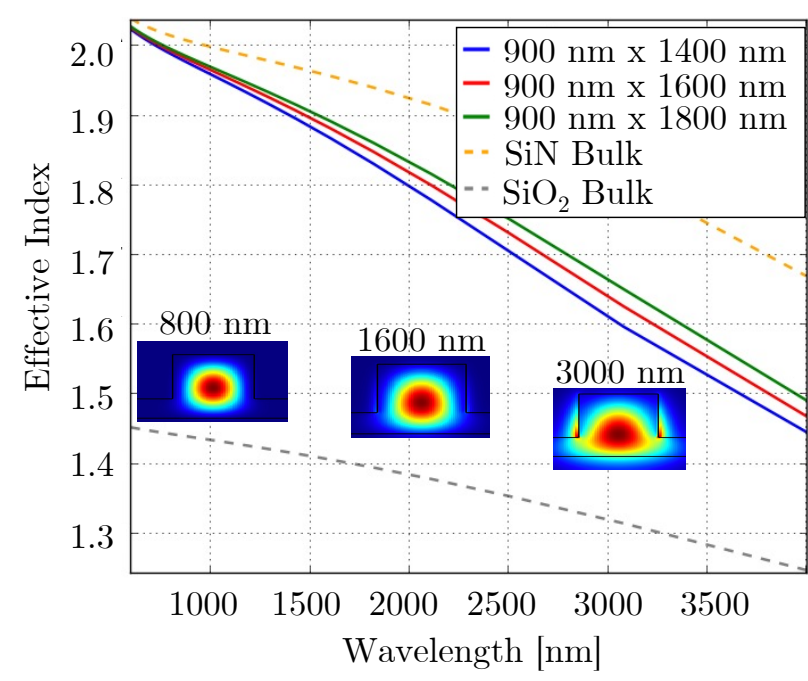

(a)

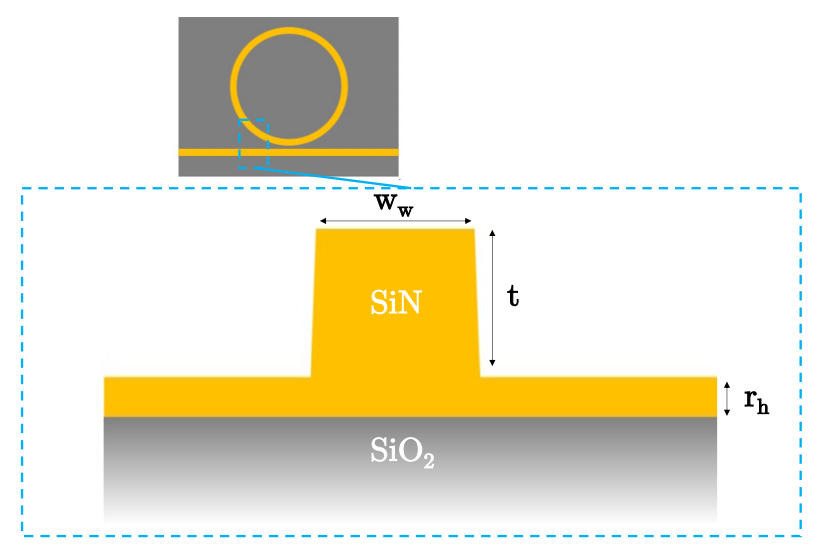

(b)

Figure 1: (a) Refractive index for bulk $\mathrm{SiN}$ and $\mathrm{SiO}_{2}$ (dashed lines) and the effective index predicted for various $\mathrm{SiN}$ waveguide cross-sections with silica cladding. The theoretically predicted intensity distributions at the fundamental mode at different wavelengths are also shown. (b) Illustration of the SiN waveguide cross-section and the microring resonator investigated in this paper.

The approach 'top-down' implemented in this paper relies on accurate nonlinear simulation tools, where the dispersion engineering properties of the waveguides are correlated to the cavity dynamics via a grey-box approach. While keeping a clear mapping of the "input vs output", "waveguide geometry vs soliton formation and characteristics", a deeper knowledge of the fabrication tolerance understanding can be achieved. While in previous works, the design of the waveguides has been explored in a few parameter space locations, here we map the main fabrication variability sources: namely waveguide thickness, waveguide width and ridge height. We first investigated the dispersion properties of the waveguides in a large parameter space. Then, the results obtained are utilised to simulate the cavity dynamics via the LLE. In absence of fabrication variability and simplifying the requirements, the region of the parameter space with negative group velocity dispersion (GVD) at the pumping wavelength favours the soliton formation. This assumption remain valid when the actual LLE simulation is carried out (see Figure 5a). In presence of fabrication variability, the actual waveguide cross-section will be different from the designed one depending of its position on the wafer and on the reticle. However, with a proper fabrication variability analysis, not only can the yield of a specific cross-section be known a priori, but can also be used as a means to take more robust design choices. For instance, enabling the decision to be in a higher yield region of the parameter space.

\subsection{Fabrication Variability in Silicon Nitride on Insulator Photonics}

Wafer fabrication of SiN on insulator PICs has the key advantage of being compatible with CMOS fabrication facilities. ${ }^{15}$ However, there is a major problem that we are faced with: the variability across the wafer affects the optical performance of the devices. Specifically the variability which we consider are the waveguide thickness and width and the ridge height. There is a further aspect that will not be considered in this work: the refractive index of the silicon nitride. As opposed to silicon, it is process dependent: SiN exhibits a refractive index function of its different chemical composition as a consequence of the deposition technique used to realise it. ${ }^{1}$ Then, the negative GVD range was analysed but not presented in the results. This range corresponds to 
the maximum broadening which correlates to the highest energy of the single pulse. Furthermore, while the waveguide thickness is an intrinsic property of the specific wafer (it depends on the uniformity and accuracy of the thin-film deposited/bonded on the buried oxide), the linewidth and the ridge height are CMOS process dependent: more specifically the linewidth is mainly dependent on the lithography process and the ridge height on the etching process following the lithography, which is valid in the first approximation. The tolerance on the linewidth and ridge height shows great results, being of the order of $\pm 2 \mathrm{~nm}$ for silicon. The thickness of the waveguide, depending on the specific wafer, has in general centimeter length scale, while the linewidth and ridge height, being dependant on photolithography, specific pattern and pattern density, have a much shorter length scale. ${ }^{16}$ The length scale and specific behavioural pattern of fabrication tolerances will not be addressed in this work, but rather combined in favour of a cumulative design optimisation.

These aspects are in general accounted for during the design phase: thanks to the maturity of silicon photonics, most of the simulation tools provide some fabrication statistics analysis. ${ }^{12}$ The complexity of the KCG design arises from the nonlinear time dynamic behind its working regime. However, the fabrication statistics tool is extremely powerful for linear circuits, which are of little to no use for the specific case. Our approach, once associated with a fabrication facility/routine, allows the mapping of the safe design regions for maximising the yield of the KCGs.

\subsection{Tolerant Design Methodology}

The methodology utilised in this work is represented in Figure 2. While the solid lines describe the standard method to the simulation of KCGs, the dashed lines describe the iteration step here proposed where the fabrication tolerances are accounted for and the low yield regions are excluded by the set of viable design parameters. The standard design procedure requires the simulation of the waveguide dispersion properties in order to find the waveguide cross-sections providing the desired dispersion characteristics. Then, dispersion engineering is carried out: several cross-sections are investigated in order to find an (often local) optimum. The dashed lines in the figure correspond to our contribution to the tolerant design methodology. Two yield analyses steps were added in this design: the first takes place after the eigenmode simulation and the second after the LLE simulation. These analyses are carried out simultaneously in order to provide details on the fabrication tolerance.

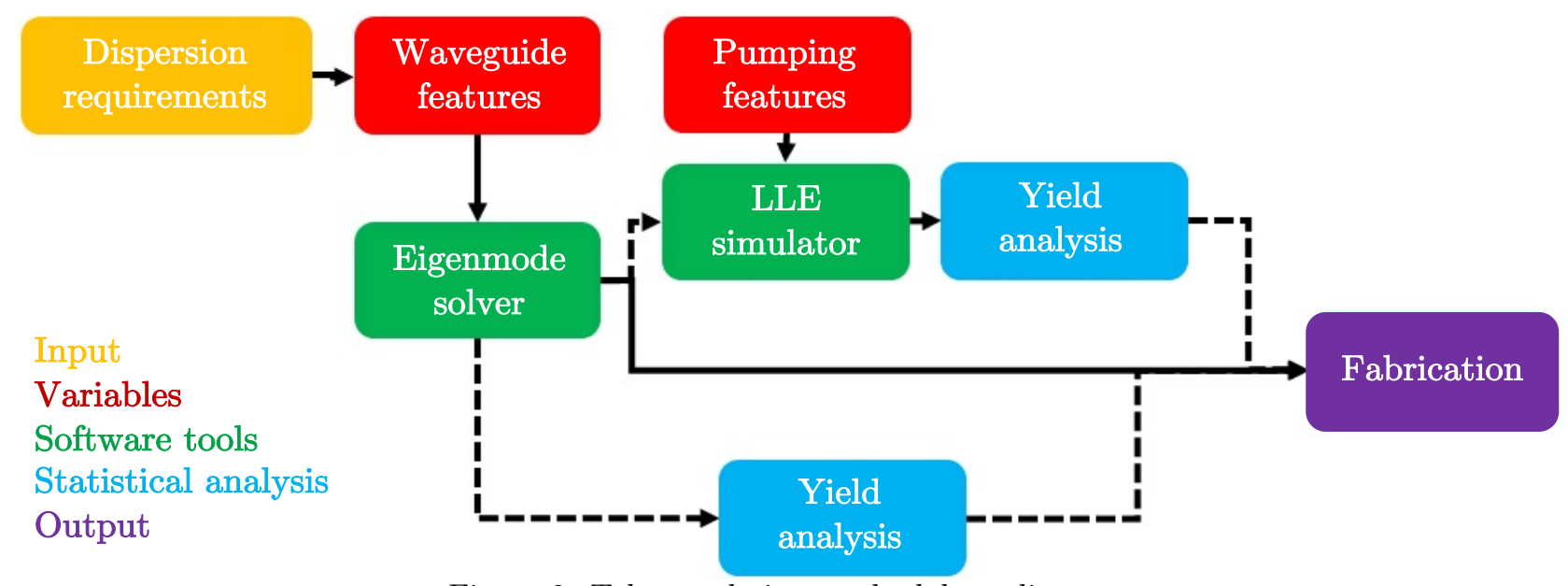

Figure 2: Tolerant design methodology diagram.

The pump wavelength is chosen to be $1550 \mathrm{~nm}$ in order to comply with standards for telecommunication applications. The pump power used for the LLE simulation is $300 \mathrm{~mW}$ since this value is often used in literature ${ }^{17}$ and it is a moderate power which induces a non-destructive power density for the device under test. The quality factor used is $10^{6}$, which is also a reasonable value for the proposed platform. ${ }^{17}$ The radius selected for the ring is $60 \mu \mathrm{m}$. The effective nonlinear coefficient at the pump frequency is $\gamma=1.55 \mathrm{~W}^{-1} \mathrm{~m}^{-1}$. It takes into account the area of the mode (the confinement factor) and the nonlinear coefficient of the material. The simulation 
exploration spanned a large parameter space including strip and ridge structures and several SiN thin-film thicknesses. The estimated waveguide propagation and coupler losses were taken into account in the simulation. The critically coupled condition was used for the simulation, therefore a self-coupling coefficient of approximately $99 \%$ was selected. Table 1 summarises these parameters.

\begin{tabular}{|l|l|}
\hline Simulation parameters & Values with corresponding units \\
\hline Pump power & $300 \mathrm{~mW}$ \\
\hline Pump wavelength & $1550 \mathrm{~nm}$ \\
\hline Q factor & $10^{6}$ \\
\hline Microring resonator radius & $60 \mu \mathrm{m}$ \\
\hline Effective nonlinear coefficient & $\gamma=1.55 \mathrm{~W}^{-1} \mathrm{~m}^{-1}$ \\
\hline Self-coupling coefficient & 0.99 \\
\hline
\end{tabular}

Table 1: LLE simulation inputs.

Figures $3 \mathrm{a}$ and $3 \mathrm{~b}$ show two of the main dispersion properties under investigation: the minimum GVD wavelength and its corresponding value, respectively.

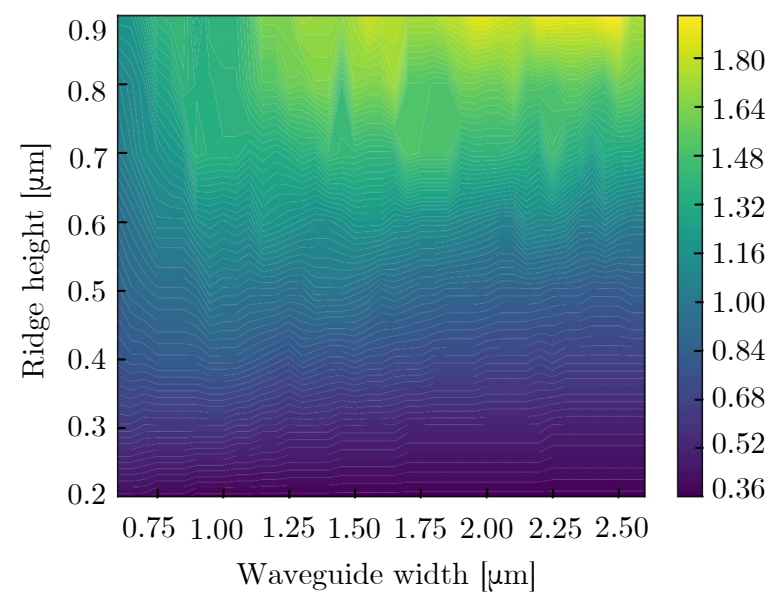

(a)

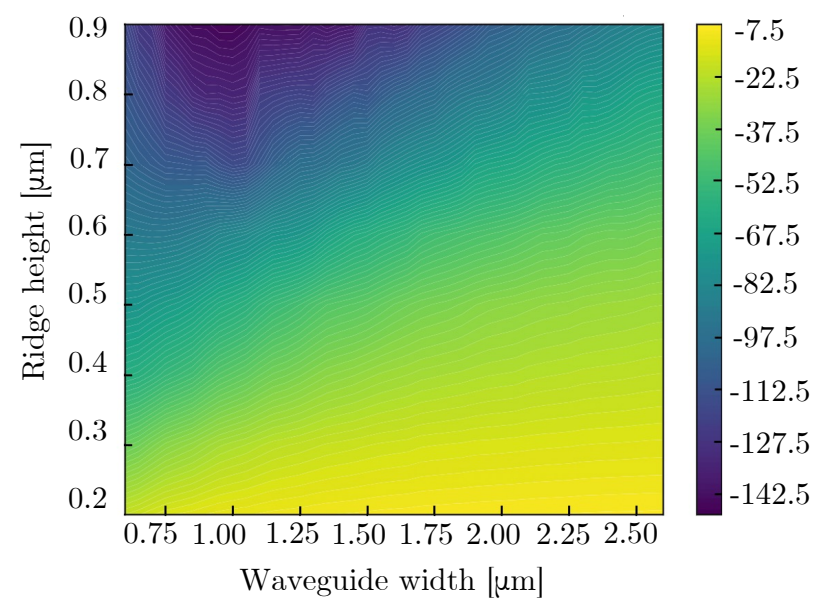

(b)

Figure 3: Surface plots illustrating (a) the minimum GVD wavelengths $[\mu \mathrm{m}]$ (b) the minimum GVD value $[\mathrm{ps} / \mathrm{nm} / \mathrm{km}]$ for various $\mathrm{SiN}$ cross-sections for a $900 \mathrm{~nm}$ thickness.

Ideally, we would want to work in the regions where the GVD is negative but in the zero proximity, because there is a performance degradation in the regions where there is a largely negative GVD value. From Figure 3b, it is shown that the negative GVD value reaches, for instance, a low of $-142 \mathrm{ps} / \mathrm{nm} / \mathrm{km}$ for an SiN cross-section with a $0.8 \mu \mathrm{m}$ ridge height and a $1 \mu \mathrm{m}$ waveguide width. Later on, this degradation in performance is confirmed in Figure 5a, illustrating the soliton number in the cavity. In fact, in that specified region, the soliton number is lower as there is a low amount of energy. Once the dispersion properties have been obtained and the related dispersion engineering has been carried out, the data is fed to the LLE where the cavity dynamics are simulated. Figure 4a illustrates the power of the single soliton state (orange solid line) and of the double soliton state (blue solid line)inside the cavity. In fact, the cavity starts with two solitons and stabilises itself to a single soliton state. Figure $4 \mathrm{~b}$ confirms this observation as it shows the single (orange solid line) and double soliton states (blue solid line) through the illustration of the electromagnetic envelope of the time-domain profile of the soliton states inside the microring resonator. 


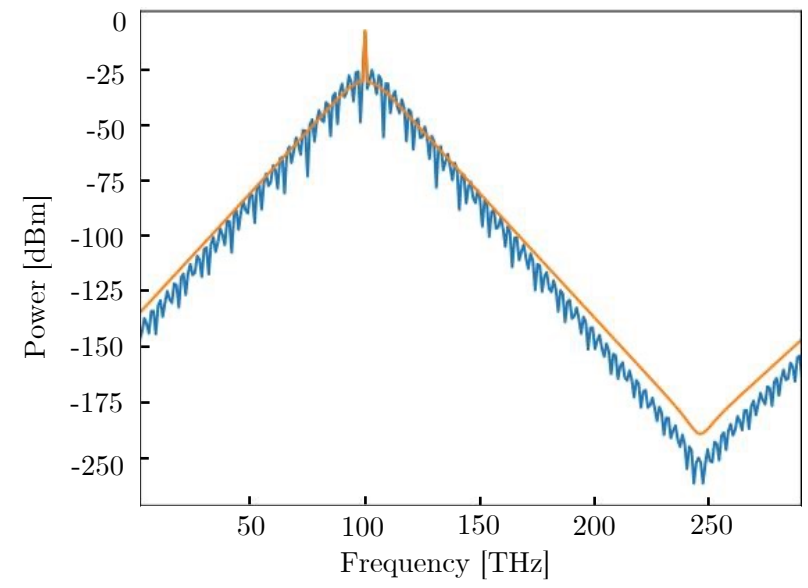

(a)

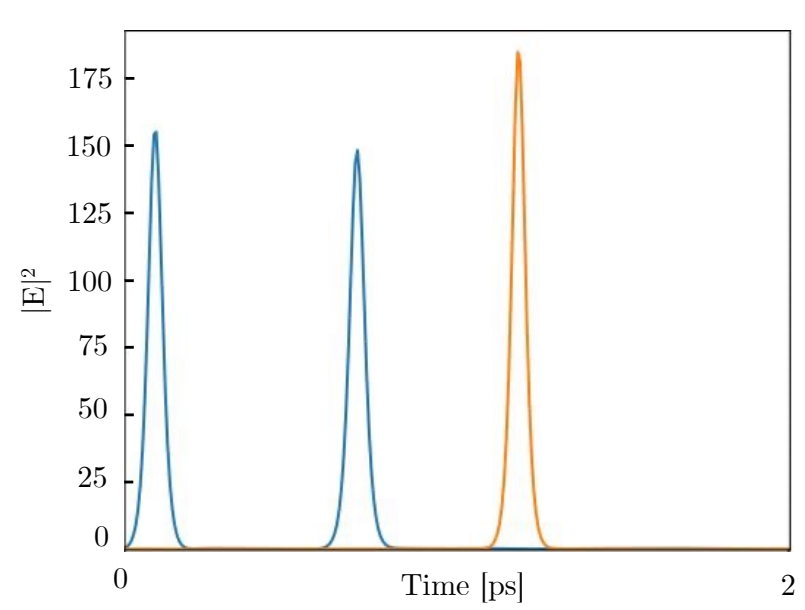

(b)

Figure 4: (a) Spectrum of the soliton combs inside the cavity: the single soliton state (orange solid line) and the double soliton state (blue solid line). (b) Electromagnetic envelope of the time-domain profile of the single (orange solid line) and double (blue solid line) soliton states inside the microring resonator.

The following information can be extracted from the data in Figure 4b: the pulse duration and broadening and the pulse energy peak, which are related to the full width at half maximum (FWHM) of the pulse. They are all of interest to various users depending on the desired application of the integrated KCG. Figures 5a and 5b illustrate two characteristics of the cavity dynamics: the number of solitons formed in the cavity and the pulse energy peak, respectively. The latter is of interest in the optical communication community as it provides an insight into the amount of power that can be transmitted down the optical fibre, although this power also is dependent on the amount of comb lines that are available for use in the optical communication system.

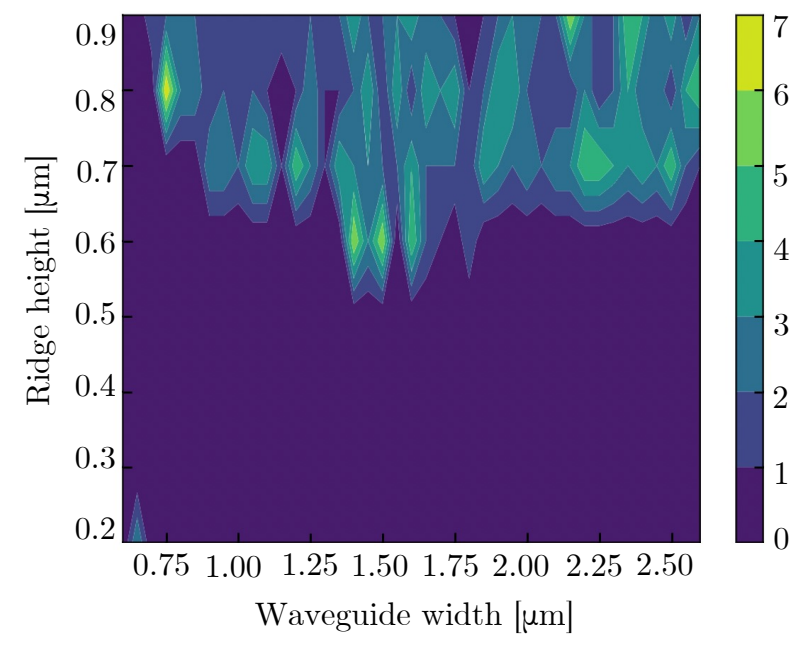

(a)

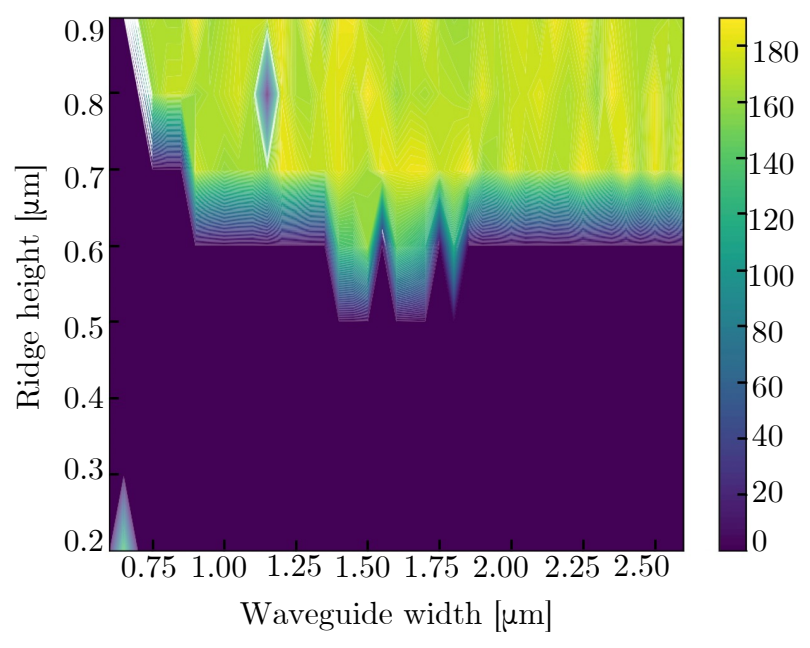

(b)

Figure 5: (a) Surface plots illustrating the number of solitons (b) and the pulse energy peak [pJ] inside the microring resonator for various SiN cross-sections for a $900 \mathrm{~nm}$ thickness.

As seen, there is a clear illustration of the regions where the design is more robust, meaning there is a higher probability of finding a soliton for cross-sections that in theory allow the soliton state, as well as a desired higher pulse energy peak. This region indicates that the larger the SiN waveguide cross-sections the higher the probability of finding a soliton and to have a higher pulse energy peak. Even if part of the parameter set excluded 
provide stable solitons in the simulation, they are kept out in order to increase the large-scale yield. The level of conservativeness can be set a priori to designing the waveguides such that the stochastic distribution of the fabrication uncertainty is systematically excluded from the selected cross-section.

\section{ANALYSIS OF FABRICATION TOLERANCE}

Since CMOS designs come with tolerances, our approach is to scale up the robustness. All designs do not come with the same safety buffer. In order to illustrate this, we will present the robustness of the designs using statistical distributions of the waveguide widths and heights to show the fabrication variability. In order to represent the "safe" regions with the presence of solitons, we define a continuous normalised Gaussian distribution in equation 1 , where $w_{w}$ and $r_{h}$ represent the width and the ridge height of the selected cross-section, respectively. Then, $w_{\min }, r_{\min } w_{\max } r_{\max }$ represent the lower and upper limits of the parameter space associated with the waveguide feature. $\sigma_{w}$ and $\sigma_{r}$ are the square roots of the fabrication variances and $S(w, r)$ is the Boolean matrix providing information on the soliton formation with respect to the waveguide's cross-section according to Figure 5 a. It is important to specify that this is an $n$ dimensional space, but we limited ourselves to a two-dimensional space for the simulation. Since the parameter space is a finite domain, the methodology carries a normalisation error, which is $\epsilon \approx 75 \%$ for any permutation of $\left(w_{w}, r_{h}\right)=\left(w_{\max , \min }, r_{\max , \min }\right)$, dropping to $\epsilon \approx 30 \%$ for any permutation of $\left(w_{w}, r_{h}\right)=\left(\left(w_{\max }-\sigma_{w}, w_{\min }+\sigma_{w}\right),\left(r_{\max }-\sigma_{r}, r_{\min }+\sigma_{r}\right)\right)$, dropping again to $\epsilon \approx 5 \%$ for any permutation of $\left(w_{w}, r_{h}\right)=\left(\left(w_{\max }-2 \sigma_{w}, w_{\min }+2 \sigma_{w}\right),\left(r_{\max }-2 \sigma_{r}, r_{\min }+2 \sigma_{r}\right)\right)$.

$$
Y_{\text {soliton }}\left(w_{w}, r_{h}\right)=\iint_{w_{\min }, r_{\min }}^{w_{\max }, r_{\max }} \frac{1}{2 \pi \sigma_{w} \sigma_{r}} S(w, r) e^{-\frac{1}{2}\left(\left(\frac{w_{w}-\mu_{w}}{\sigma_{w}}\right)^{2}+\left(\frac{r_{h}-\mu_{r}}{\sigma_{r}}\right)^{2}\right)} d w d r
$$

Therefore, it is possible to quantify the yield at every point on the surface plots representing the dispersion properties of the SiN waveguides, as well as on the plots of the cavity dynamics, after the LLE simulations. The results in Figures $6 \mathrm{a}$ and $6 \mathrm{~b}$ use equation 1 in order to quantify the yield for the various SiN waveguide cross-sections in the case where the negative GVD value is smaller than $-20 \mathrm{ps} / \mathrm{nm} / \mathrm{km}$, and then for whether there is soliton formation in the cavity, respectively. To obtain these results, a tolerance of $20 \mathrm{~nm}$ was set: more specifically, $20 \mathrm{~nm} \sigma_{w}$ and $\sigma_{r}$, representing the square roots of the variance, respectively, for the waveguide width and the ridge height. The assumption that the variance is independent on the specific value, has also been made (e.g.the variance of the waveguide width does not depend on the target width).

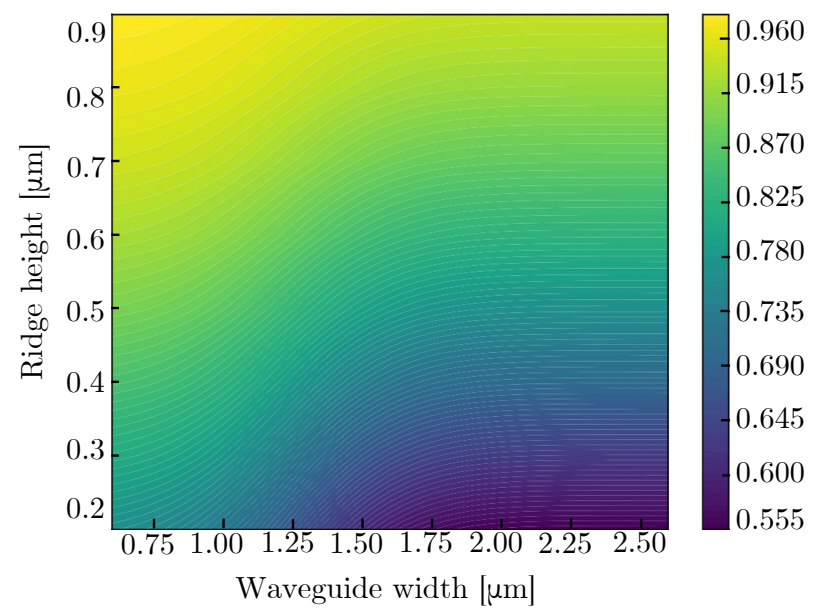

(a)

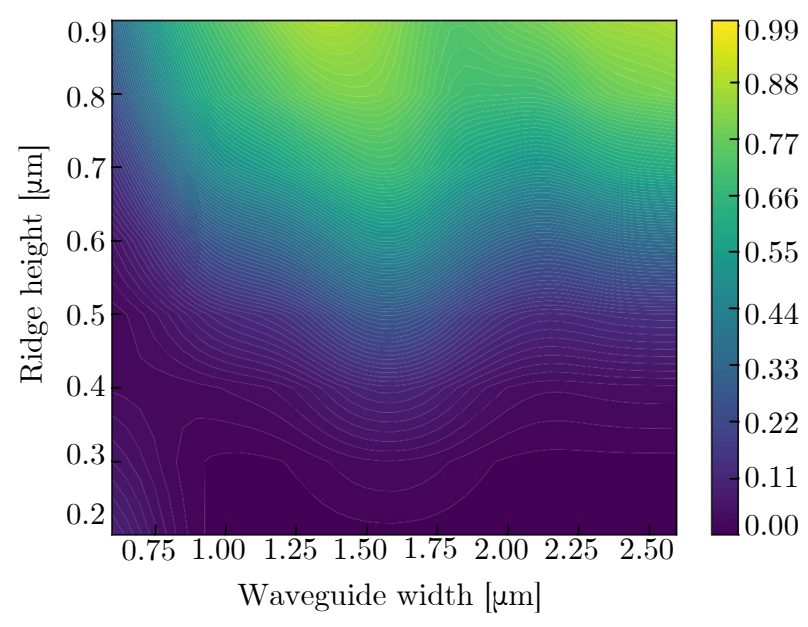

(b)

Figure 6: (a) Yield for a minimum GVD value $[\mathrm{ps} / \mathrm{nm} / \mathrm{km}$ ] smaller than $-20 \mathrm{ps} / \mathrm{nm} / \mathrm{km}$ (b) and a soliton formation for $\mathrm{a} \pm 20 \mathrm{~nm}$ tolerance for the waveguide width and ridge height.

These figures enable us to identify the regions where the design exhibits the largest robustness, in which we are most likely to find solitons across dies and across wafers. In fact, Figure $6 \mathrm{~b}$ shows a yield of approximately 
$99 \%$ for a few areas on the surface plot: these areas correspond to SiN waveguides with top waveguide widths, $w_{w}$, from approximately 1250 to $1550 \mathrm{~nm}$, and with a ridge height, $r_{h}$, from approximately 800 to $900 \mathrm{~nm}$. In these areas, we are most likely to have a robust design. In fact, this is in agreement with Figure 6a which shows the yield for a minimum GVD value that is smaller than $-20 \mathrm{ps} / \mathrm{km} / \mathrm{km}$. In fact, the larger cross-sections, with ridge heights, $r_{h}$, greater than $800 \mathrm{~nm}$, show a yield of approximately $96 \%$. Therefore, these plots can be used as a powerful predictive tool for users seeking to increase their yield.

\section{CONCLUSION}

Fabrication variability is still an open problem affecting integrated CMOS compatible photonics, which is even more pronounced when working with SiN on insulator due to its lower maturity. While several techniques to improve the yield of linear components have been proposed, nonlinear devices still suffer reprehensibility across the wafer(s). We proposed and tested a fabrication variability aware design strategy which enables the prediction of the KCG yield. The methodology can be utilised at any stage of the design flow, since the yield prediction can be obtained while simulating the optical properties of the waveguide's cross-section and/or when simulating the actual KCG. Nevertheless, the methodology can be strengthened even further in the presence of experimental data. We tested the procedure to predict the yield of the GVD of the waveguides as well as the yield of the soliton generation. The latter presented itself as a powerful tool towards the yield maximisation of KCG on SiN on insulator platforms: the yield mapping via LLE simulation can provide a powerful tool toward mass production KCGs by means of the selection of the design parameters with the highest predicted yield.

\section{ACKNOWLEDGMENTS}

The authors would like to acknowledge the Engineering and Physical Sciences Research Council (EPSRC) for financial support (No. EP/S022139/1).

\section{REFERENCES}

[1] Rahim, A., Ryckeboer, E., Subramanian, A. Z., Clemmen, S., Kuyken, B., Dhakal, A., Raza, A., Hermans, A., Muneeb, M., Dhoore, S., Li, Y., Dave, U., Bienstman, P., Thomas, N. L., Roelkens, G., Thourhout, D. V., Helin, P., Severi, S., Rottenberg, X., and Baets, R., "Expanding the silicon photonics portfolio with silicon nitride photonic integrated circuits," J. Lightwave Technol. 35, 639-649 (Feb 2017).

[2] Dwivedi, S., Ruocco, A., Vanslembrouck, M., Spuesens, T., Bienstman, P., Dumon, P., Vaerenbergh, T. V., and Bogaerts, W., "Experimental extraction of effective refractive index and thermo-optic coefficients of silicon-on-insulator waveguides using interferometers," J. Lightwave Technol. 33, 4471-4477 (Nov 2015).

[3] Shiramin, L. A. and Van Thourhout, D., "Graphene modulators and switches integrated on silicon and silicon nitride waveguide," IEEE Journal of Selected Topics in Quantum Electronics 23(1), 94-100 (2016).

[4] Wang, C., Zhang, M., Yu, M., Zhu, R., Hu, H., and Loncar, M., "Monolithic lithium niobate photonic circuits for kerr frequency comb generation and modulation," Nature communications 10(1), 1-6 (2019).

[5] Wang, L., Chang, L., Volet, N., Pfeiffer, M. H., Zervas, M., Guo, H., Kippenberg, T. J., and Bowers, J. E., "Frequency comb generation in the green using silicon nitride microresonators," Laser 83 Photonics Reviews 10(4), 631-638 (2016).

[6] Bogaerts, W., De Heyn, P., Van Vaerenbergh, T., De Vos, K., Kumar Selvaraja, S., Claes, T., Dumon, P., Bienstman, P., Van Thourhout, D., and Baets, R., "Silicon microring resonators," Laser 8 Photonics Reviews 6(1), 47-73 (2012).

[7] Lugiato, L. A., Prati, F., Gorodetsky, M. L., and Kippenberg, T. J., "From the lugiato\&\#x2013;lefever equation to microresonator-based soliton kerr frequency combs," Philosophical Transactions of the Royal Society A: Mathematical, Physical and Engineering Sciences 376(2135), 20180113 (2018).

[8] Gaeta, A. L., Lipson, M., and Kippenberg, T. J., "Photonic-chip-based frequency combs," Nature Photonics 13(3), 158-169 (2019).

[9] Moille, G., Li, Q., Lu, X., and Srinivasan, K., "pylle: a fast and user friendly lugiato-lefever equation solver," arXiv preprint arXiv:1903.10441 (2019). 
[10] Qi, Y. and Li, Y., "Integrated lithium niobate photonics," Nanophotonics 1(ahead-of-print) (2020).

[11] Ruocco, A., Fiers, M., Vanslembrouck, M., Vaerenbergh, T. V., and Bogaerts, W., "Multi-parameter extraction from SOI photonic integrated circuits using circuit simulation and evolutionary algorithms," in [Smart Photonic and Optoelectronic Integrated Circuits XVII], Eldada, L. A., Lee, E.-H., and He, S., eds., 9366, 1 - 9, International Society for Optics and Photonics, SPIE (2015).

[12] Lu, Z., Jhoja, J., Klein, J., Wang, X., Liu, A., Flueckiger, J., Pond, J., and Chrostowski, L., "Performance prediction for silicon photonics integrated circuits with layout-dependent correlated manufacturing variability," Optics express 25(9), 9712-9733 (2017).

[13] Bogaerts, W. and Chrostowski, L., "Silicon photonics circuit design: methods, tools and challenges," Laser ES Photonics Reviews 12(4), 1700237 (2018).

[14] Krasnokutska, I., Tambasco, J.-L. J., and Peruzzo, A., "Tunable large free spectral range microring resonators in lithium niobate on insulator," Scientific reports 9(1), 1-7 (2019).

[15] Moss, D. J., Morandotti, R., Gaeta, A. L., and Lipson, M., "New cmos-compatible platforms based on silicon nitride and hydex for nonlinear optics," Nature photonics 7(8), 597-607 (2013).

[16] Selvaraja, S. K., Bogaerts, W., Dumon, P., Van Thourhout, D., and Baets, R., "Subnanometer linewidth uniformity in silicon nanophotonic waveguide devices using cmos fabrication technology," IEEE Journal of Selected Topics in Quantum Electronics 16(1), 316-324 (2009).

[17] Wang, C., Zhang, M., Yu, M., Zhu, R., Hu, H., and Loncar, M., "Monolithic lithium niobate photonic circuits for kerr frequency comb generation and modulation," Nature communications 10(1), 1-6 (2019). 